\title{
REVIEW
}

\section{The epididymis, cytoplasmic droplets and male fertility}

\author{
Trevor G Cooper
}

The potential of spermatozoa to become motile during post-testicular maturation, and the relationship between the cytoplasmic droplet and fertilizing capacity are reviewed. Post-testicular maturation of spermatozoa involves the autonomous induction of motility, which can occur in vivo in testes with occluded excurrent ducts and in vitro in testicular explants, and artefactual changes in morphology that appear to occur in the testis in vitro. Both modifications may reflect time-dependent oxidation of disulphide bonds of head and tail proteins. Regulatory volume decrease (RVD), which counters sperm swelling at ejaculation, is discussed in relation to loss of cytoplasmic droplets and consequences for fertility. It is postulated that: (i) fertile males possess spermatozoa with sufficient osmolytes to drive RVD at ejaculation, permitting the droplet to round up and pinch off without membrane rupture; and (ii) infertile males possess spermatozoa with insufficient osmolytes so that RVD is inadequate, the droplet swells and the resulting flagellar angulation prevents droplet loss. Droplet retention at ejaculation is a harbinger of infertility caused by failure of the spermatozoon to negotiate the uterotubal junction or mucous and reach the egg. In this hypothesis, the epididymis regulates fertility indirectly by the extent of osmolyte provision to spermatozoa, which influences RVD and therefore droplet loss. Man is an exception, because ejaculated human spermatozoa retain their droplets. This may reflect their short midpiece, approximating head length, permitting a swollen droplet to extend along the entire midpiece; this not only obviates droplet migration and flagellar angulation but also hampers droplet loss.

Asian Journal of Andrology (2011) 13, 130-138; doi:10.1038/aja.2010.97; published online 15 November 2010

Keywords: epididymis; fertility; infertility; sperm maturation

\section{INTRODUCTION}

During passage of mammalian spermatozoa through the epididymal duct, the functionally incompetent germ cell produced by the testis is matured and stored. In this time (around 1-2 weeks in most species), the spermatozoon undergoes many changes that prepare it for achieving the diverse tasks required of it. At ejaculation, when it is rapidly expelled from the epididymis through the vas deferens to the world outside, the spermatozoon undergoes another phase in which it encounters fluids of the male accessory sex glands, escapes from them in the vagina, cervix or uterus, depending on species, and interacts with the oviductal lining before fertilizing the female gamete. Many mechanisms control the timing of these events, all of which require adequate responses by the fertilizing spermatozoon, and several of these have their origins in the epididymis. This paper addresses a few controversial, novel or still unanswered topics related to the maturation, volume regulation and morphology of spermatozoa.

\section{SPERM MOTILITY AND MORPHOLOGY}

Changes occur in the epididymis to what appear to be anatomically complete spermatozoa shed from the seminiferous tubule. Both sperm appearance and motion develop into the characteristics of mature spermatozoa stored in the cauda epididymidis. These developments occur under the influence of epididymal secretions. If spermiogenesis is defective, the shed spermatozoon may be unable to respond adequately to the epididymal environment.
Potential for sperm motility in the testis

To monitor the motility of spermatozoa, it is routine to transfer them to incubation medium containing metabolic substrates and an ionic environment similar to that found within the female tract. This mimics the abrupt changes at ejaculation that activates the potentially motile mature spermatozoa held quiescent in the cauda epididymidis. In contrast, spermatozoa removed from the testis are usually immotile, and their coordinated ability to move develops in the epididymis at a location that is species dependent. ${ }^{1}$ Thus, spermatozoa obtained by mincing human testicular biopsy are usually immotile, although motile cells can be obtained from the testis if its excurrent duct system is blocked. ${ }^{2}$ This apparent precocious development of motility could represent the effects of epididymal secretions normally involved in the development of motility within the epididymis that have back-fluxed into the testis under the abnormal conditions of ductal occlusion. It is more likely, however, that spermatozoa have inherently acquired some motility, independent of the epididymis, as spermatozoa trapped in epididymal spermatocoeles, which contain no epididymal secretions, ${ }^{3}$ are poorly motile, although a few can fertilize eggs in vitro. ${ }^{4}$

Observations made in in vitro fertilization clinics indicate that increased numbers of progressively motile spermatozoa can be recovered from testicular explants by their mere incubation for up to 3 days in vitro (Figure 1), ${ }^{5-8}$ in the complete absence of epididymal secretions. Clinical advantage of this is routinely taken to increase the number of spermatozoa available for intracytoplasmic sperm injection. The nature 


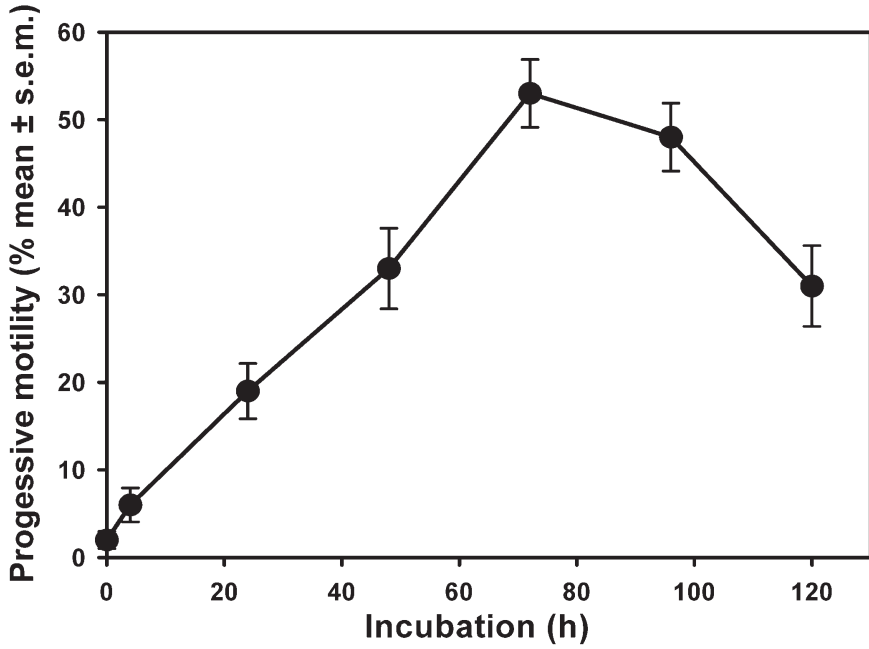

Figure 1 The percentage of spermatozoa exhibiting progressive motility (ordinate) at various times (abscissa) after retention within human testicular implants. Data redrawn from Liu et al. ${ }^{8}$ with permission.

of this activation of inherent motility has been neither explained nor investigated, but in the absence of any epididymal tissue, a timedependent acquisition of motility potential must be considered. There is a precedent for this in that ovine rete testicular spermatozoa, when stored within their native fluid, develop some capacity for movement. ${ }^{9}$

\section{Changes in motility potential during epididymal transit}

Spermatozoa are thought to be largely immotile within the epididymal canal, which is technically difficult to assess: in the proximal regions as a consequence of their immaturity, but in the distal regions-once the capacity for motility has been acquired-it is enforced by low $\mathrm{pH}$, low concentrations of sodium $\left(\mathrm{Na}^{+}\right)$and high concentrations of potassium $\left(\mathrm{K}^{+}\right)$or the presence of viscous mucoproteins, again depending on species. ${ }^{10}$ Some sperm motility can be observed in undiluted bovine epididymal luminal contents observed under paraffin oil, ${ }^{11}$ but the motility of these distal spermatozoa is fully initiated (the spermatozoa become 'activated') once they are released from this environment and placed in medium approximating that of the female tract. As distal epididymal contents are hypertonic to female tract fluids, ${ }^{12}$ transfer of mature epididymal spermatozoa to media of composition mimicking that of female tract fluids will encourage hypotonic swelling. This is currently thought to be countered by volume regulation, accompanied by entry of external $\mathrm{Na}^{+}$, driving $\mathrm{H}^{+}$out via the sodium-hydrogen exchanger. This raises intracellular $\mathrm{pH}$, which activates axonemal sliding that is inhibited by low $\mathrm{pH}^{13}$ and activates $\mathrm{K}^{+}$ channels that are responsible for hyperactivation. ${ }^{14,15}$

Immature spermatozoa from the caput epididymidis, with their less developed axonemal apparatus, will not respond in the same way as mature cells, and testicular spermatozoa, where the change in $\mathrm{K}^{+}$ gradient and the osmotic challenge are smaller, do not respond to this medium by being motile. Thus, the immediate environment only permits the expression of motility in the cells that have developed sufficiently to be able to respond to the physiological triggers. This process involves development of the response to $\mathrm{Ca}^{2+}$ and cyclic AMP and downstream signalling systems. ${ }^{13}$

\section{Apparent changes in sperm head morphology in the testis}

An interesting clinical observation that more human testicular spermatozoa with normal morphology are seen in testicular biopsies after

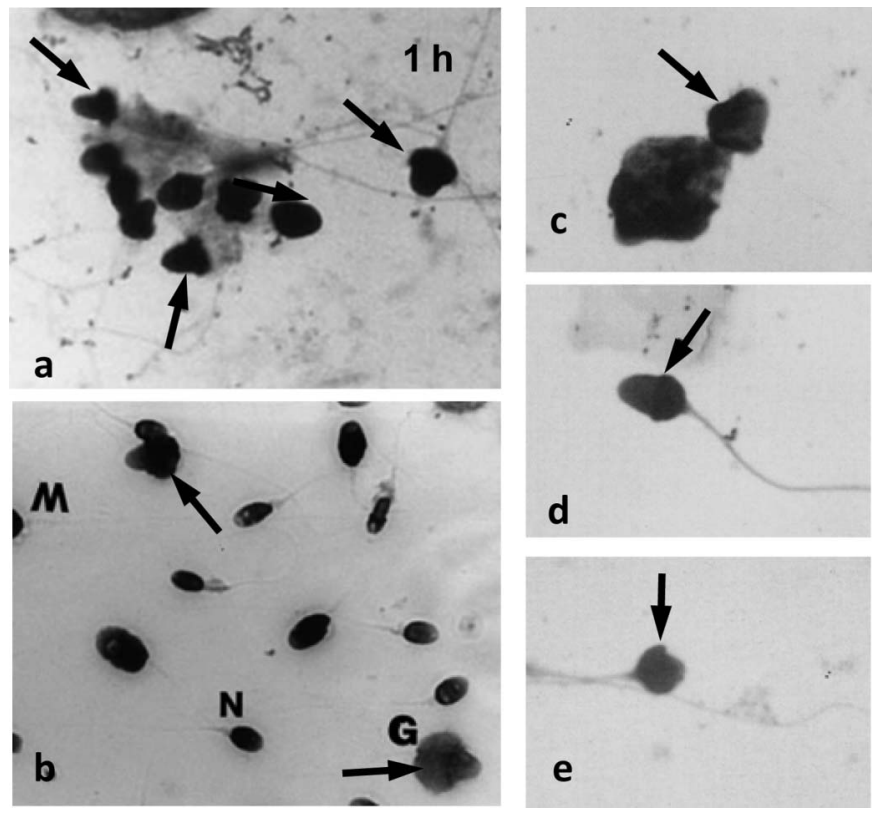

Figure 2 Montage of micrographs of spermatozoa that display post-acrosomal expansion (arrows). (a) Testicular spermatozoa from explants (Liu et al. ${ }^{8}$ with permission), (b) epididymal spermatozoa (Soler et al. ${ }^{17}$ with permission) and (ce) seminal spermatozoa (Ludwig and Frick ${ }^{24}$ with permission). The ejaculated spermatozoa resemble the immature testicular and caput epididymidal spermatozoa in air-dried smears, and thus may indicate epididymal dysfunction (inadequate maturation or unusually rapid epididymal transit). Sperm head forms: G, ghosts; M, macro; N, normal.

$72 \mathrm{~h}$ in vitro than in the original testicular explants ${ }^{8}$ (Figure 2a), has never received the comment it deserves, although this change parallels the in vitro, epididymal-independent development of progressive motility. Morphology was examined after the routine seminal procedure of staining fixed, air-dried smears, a process that subjects the spermatozoa not only to shear forces during smearing but also to hyperosmotic insults during fluid evaporation and presumed cellular dehydration before the cells are fixed, rehydrated, stained and observed. This procedure is known to produce 'exploded' postacrosomal regions (producing 'acorn-shaped' sperm heads) of unfixed, immature, primate caput epididymidal spermatozoa (Figure 2b). The fact that these forms are never observed if the cells are fixed before air drying and staining ${ }^{16}$ demonstrates that the abnormal sperm heads are artefacts of the air-drying method and do not reflect the morphology of the living cells within the epididymis.

The abnormal morphologies of unfixed, air-dried human testicular spermatozoa seen 1 day after excision of the tissue are just those with the swollen post-acrosomal areas observed in unfixed immature monkey spermatozoa. Furthermore, it was these 'acorn-shaped' forms that were no longer observed in the air-dried specimens prepared $72 \mathrm{~h}$ after culture, leading to the conclusion that 'normal' forms had somehow appeared, developed or matured on mere incubation within testicular tissue. ${ }^{8}$ A more logical conclusion can be drawn from the observations that the abnormal human 'acorn' sperm head forms observed in unfixed, air-dried, immature caput epididymidal spermatozoa are not seen in preparations of unfixed, air-dried, mature human epididymal spermatozoa. ${ }^{16,17}$ These observations suggest that the mature cells develop a maturation-dependent resistance to the forces of air drying within the epididymis. In the same way, the testicular spermatozoa, aged in vitro in the explants, have most likely developed in 
vitro a similar resistance to cellular distortion from air drying-created osmotic forces. In both instances, this is probably due to the intracellular oxidation of structural sulphydryl proteins, ${ }^{18,19}$ which may also have promoted flagellar stiffness in vitro that would aid the (still unexplained) initiation of motility.

\section{Changes in sperm morphology during epididymal transit}

Within the epididymis, spermatozoa also undergo changes in their morphology, notably that of their head and their cytoplasm. When samples from rats ${ }^{20}$ and hamsters ${ }^{21}$ are initially fixed before examination, and are thus likely to represent the status of spermatozoa in situ at the time of fixation, a fine tuning of the shape of the sperm head is reflected in a decline in total sperm head area and change in angle of curvature of the acrosomal hook. The hook-shaped form of the rodent sperm head has been implicated in formation of sperm clusters that aid sperm motion en masse in some species, ${ }^{22}$ but the sickle-shaped form could also be instrumental in permitting the single-file movement of individual spermatozoa through the interdigitating epithelial folds of the uterotubal junction post-coitum, seen in histological sections from the mouse. ${ }^{23}$ Maturational changes in the angle of the acrosomal hook may benefit negotiation of the female tract by the spermatozoon in those species in which the uterotubal barrier is the main obstacle to access to the egg.

Computer-aided analysis of human sperm head morphometry has demonstrated that the length/width ratio of spermatozoa with 'normal' appearance (that is, not 'exploded' heads) decreases as spermatozoa are taken from more distal regions of the epididymis. ${ }^{17}$ Unlike the rodent samples, however, the human spermatozoa were prepared in the manner routine for human seminal spermatozoa (air drying), known to induce artefactual swelling of immature sperm heads (Figure 2b). These apparently 'maturational' changes in testicular tissue may, again, not truly reflect physiological changes occurring to maturing spermatozoa in vivo, but rather be demonstrating a different response of mature and immature spermatozoa to the physical processes to which they have been subjected.

Although these morphological forms are artefacts of sperm preparation, they may provide useful clinical information, as the presence of 'acorn-shaped' sperm heads in semen (Figure 2c-e), which has been interpreted as reflecting abnormal spermatogenesis, ${ }^{24}$ may instead be demonstrating the presence of immature spermatozoa in the cauda, suggestive either of enhanced epididymal sperm transport or failed epididymal sperm maturation. In either case, abnormal seminal sperm morphology may have an epididymal cause and be worthy of extra study.

\section{CYTOPLASMIC DROPLETS: ORIGIN AND MIGRATION}

During normal spermatogenesis, most of the round spermatid's cytoplasm is phagocytosed as 'residual bodies' by the Sertoli cell at spermiogenesis, and only a small cytoplasmic residue (the 'cytoplasmic droplet') remains applied to the elongated spermatid after release from the germinal epithelium. A characteristic morphological change to spermatozoa during epididymal transit is the caudal migration of the cytoplasmic droplet away from the neck, behind the head, to the annulus, at the end of the midpiece. This was first reported by Merton $^{25}$ for the mouse and subsequently confirmed in other species. The epididymal location of this migration varies among species, ${ }^{26}$ but the physiological significance of it, if any, has yet to be defined. The plasma membrane overlying the droplet is contiguous with that of the rest of the cell so that the cytoplasm comes into close circumferential proximity to the outer mitochondrial membranes once the droplet has passed caudally. Whether this migration affects the maturational changes in lipid mobility over the mitochondria ${ }^{27,28}$ remains to be elucidated.

Another unknown mechanism is how the droplet migrates. The proximal droplets of some ovine testicular spermatozoa begin to move distally during storage in ret testis fluid ${ }^{9}$ and the unphysiological stresses of centrifugation cause the neck droplets of caprine and porcine testicular spermatozoa to shift caudally; ${ }^{29,30}$ hence, perhaps the physical jostling of highly concentrated spermatozoa being moved by epididymal peristalsis could affect the same end result in vivo. Spermatozoa are certainly physically sensitive, they can lose droplets to shearing forces ${ }^{31}$ and head-to-head contact induces coalescence of membrane lipids at the site of contact. ${ }^{32}$ It could also be that this movement is related to the volume regulation postulated to occur within the lumen (see below), necessarily centred on this organelle, the largest volume of cytoplasm the spermatozoon has. In this scenario, the water efflux that occurs during isovolumetric regulation would occur at the cephalic pole of the droplet and influx of water and osmolytes in the caudal pole, leading to a gradual caudal 'creeping' of the droplet along the midpiece (Figure 3). This speculation requires differential location of the channels mediating such fluid transport on

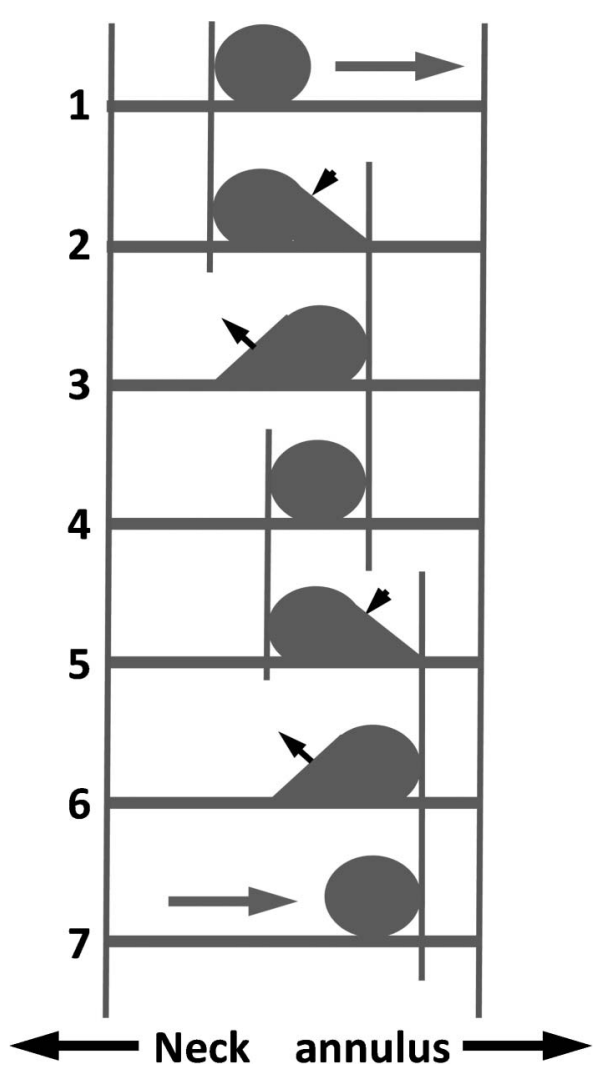

Figure 3 Schematic representation of the mode of migration of a cytoplasmic droplet along the midpiece within the epididymal lumen. Isovolumetric regulation in response to intraluminal hypertonicity is considered here to involve water efflux at the cephalic pole of the droplet (large black arrows) and water and osmolyte influx at the caudal pole (short black arrows). 1: Initial position of droplet near the neck; 2: water and osmolytes enter the caudal pole of the droplet, extending its size distally; 3 : increased droplet volume promotes water loss from the cephalic pole of the droplet, restoring its size; 4: The new more distally located droplet position; 5 and 6 repeat stages 2 and 3 above; 7: an even more distal location of the droplet. In this way, there would be a gradual distal 'creeping' (horizontal grey arrow) of the droplet from the neck towards the annulus. 
the droplet's membrane. Changes in cytoskeletal proteins may also be involved, possibly responding the continual changes in ionic strength within the droplets.

\section{POST-EPIDIDYMAL EVENTS}

Seminal emission in some species is rapid (for example, horse, bull and man) meaning that spermatozoa from the distal cauda epididymis and vas deferens are mixed with accessory gland secretions within seconds. In other species, ejaculation takes place over 20-30 min (for example, boar and dog). Either way, the spermatozoa soon come into contact with a different fluid for the second time in the female tract. In the female tract, the first (sperm- and prostatic secretion-rich) portion of the human ejaculate comes into contact with the mid-cycle cervical mucus extending into the vaginal cavity. ${ }^{33}$ The rest forms an intravaginal pool, from which spermatozoa are released as liquefaction occurs. In the laboratory, however, the entire seminal volume ejaculated is collected into one vessel, and the usually temporally separable fractions are collected together and analysed only after liquefaction has occurred and the sample thoroughly mixed. This has repercussions for the interpretation of experiments on human spermatozoa (see below).

\section{Sperm volume regulation: the fluid environment}

Spermatozoa are stored in the distal epididymis, highly concentrated in a high osmolality, high $\mathrm{K}^{+}$, low ionic strength, low $\mathrm{Na}^{+}$and low $\mathrm{pH}$ fluid. On ejaculation, they experience a reversal of most of these conditions as they enter high ionic strength fluids of low osmolality, low $\mathrm{K}^{+}$and high $\mathrm{Na}^{+}$, together with additional components from the accessory sex glands. In mice, seminal vesicle fluid is iso-osmolal with blood, ${ }^{12}$ that is, hypotonic with respect to epididymal contents. ${ }^{34}$ This means that cauda epididymidal spermatozoa are suddenly confronted at ejaculation with a drastic decrease in osmolality, initially in the accessory gland fluids, which expel them from the male tract, and later in the hypotonic female tract fluid. This would lead to an influx of water and cell swelling if this process were not countered by the regulatory volume decrease (RVD). RVD has been demonstrated to occur under physiological hypotonic stress in cauda epididymidal and ejaculated spermatozoa from several species. ${ }^{35}$

\section{Sperm volume regulation: sperm fragility}

The increasing osmosensitivity of spermatozoa and the fragility of their flagellar membranes as they migrate through the epididymis of rams, ${ }^{36,37}$ rats $^{38}$ and kangaroos ${ }^{39}$ implies a need for volume regulation if osmotic rupture of the cell is to be avoided. Caput spermatozoa from the bull ${ }^{40}$ are able to regulate volume, but an improvement in osmolyte handling is observed on epididymal maturation in this species, a change that can be effected by incubation with epididymal proteins. ${ }^{41}$ Similar observations in the rat ${ }^{42}$ suggest that the maturational process occurs more proximally in the epididymides of this species than in others.

\section{Osmolytes and sperm volume regulation}

Although osmolyte levels have not been measured in immature (caput epididymidal) spermatozoa from the monkey and mouse, which are unable to perform RVD, ${ }^{43,44}$ there are lower amounts of intracellular myo-inositol and glutamate in mature (cauda epididymidal) spermatozoa from infertile c-ros knockout (KO) mice, which are unable to control their volume, than those in the fertile heterozygous males, which can. ${ }^{45}$ These observations are consistent with a role for organic osmolytes in regulating volume in mature sperm cells. The fact that high concentrations of these osmolytes prevent sperm RVD in hypotonic media ${ }^{45}$ suggests that they do serve that role in the mouse. In man, the concentration of organic osmolytes in vas deferens fluid is lower than that in rodents (but still at $\sim 5 \mathrm{mmol}^{-1}$, a thousand times higher than that in blood) and $\mathrm{K}^{+}$is a major cation. ${ }^{46}$ It is noteworthy that raised extracellular $\mathrm{K}^{+}$is able to prevent RVD in human spermatozoa ${ }^{47}$ and $\mathrm{K}^{+}$efflux has been monitored during RVD of bovine spermatozoa. $^{48}$

\section{Source of sperm osmolytes: the epididymis?}

It has been suggested that the epididymis is involved in loading spermatozoa with these osmolytes by the process of regulatory volume increase. ${ }^{26}$ In this hypothesis, permeant osmolytes (and obliged cell water) are taken up to increase cell volume in the face of hypertonicityinduced cell shrinkage due to impermeant osmolytes such as glycerophosphocholine. In rodents, organic osmolytes are major constituents of epididymal fluid in the cauda, ${ }^{49}$ with millimolar concentrations (1000-fold higher than those in blood) of glycerophosphocholine, L-carnitine, myo-inositol and D-glutamate, some or all of which could act as osmolyte reserves for maturing spermatozoa. Certainly there are higher intracellular levels of L-carnitine and D-glutamate in porcine cauda epididymidal spermatozoa than those from the caput. ${ }^{50,51}$ The uptake of carnitine into epididymal spermatozoa from the mouse has been demonstrated, and sperm expression of the organic cation (and carnitine) transporters OCTN2 and OCTN3 increases on maturation. ${ }^{52}$ They are localized in the principal- and midpiece, respectively, but no mention of the droplets was made.

\section{Site of RVD channels: the cytoplasmic droplets}

The cytoplasmic droplet is one site of osmolytes channels involved in RVD. In the mouse, $\mathrm{K}^{+}$channels, $\mathrm{Cl}^{-}$channels and $\mathrm{K}^{+}-\mathrm{Cl}^{-}$cotransporters, and in man $\mathrm{K}^{+}$and $\mathrm{Cl}^{-}$channels, have been located there, at the neck and along the midpiece. ${ }^{35}$ Recently, water channels have been detected on the cytoplasmic droplet in mice, ${ }^{53}$ rats $^{54}$ and around midpiece membranes in man. ${ }^{55}$ These channels would become operative when spermatozoa are subjected to hypotonicity at ejaculation.

\section{Cytoplasmic droplets: post-ejaculatory loss and fertility}

Despite widespread statements to the contrary, ${ }^{56}$ the cytoplasmic droplet of spermatozoa in most species is not lost within the epididymis. ${ }^{25}$ Observations on freshly fixed luminal contents confirm this in rats, ${ }^{57}$ mice ${ }^{58}$ and other species. ${ }^{26}$ Although it is true that the whole droplet is removed by epithelial phagocytosis in the opossum, ${ }^{59}$ and the contents of ruptured droplets are phagocytosed in rats, ${ }^{60}$ in most species (but not in man; see below) the droplet remains until ejaculation. ${ }^{61-63}$ The fact that very few ejaculated spermatozoa have droplets in rams, boars, bulls and goats ${ }^{64-68}$ suggests that they are removed around the time of ejaculation. Merton ${ }^{25}$ showed that uterine spermatozoa in the mouse lacked droplets and Yeung et al. ${ }^{23}$ showed that flagella of uterine spermatozoa from fertile males are straight, whereas the uterine spermatozoa from infertile c-ros $\mathrm{KO}$ males are angulated at the site of the retained droplet.

\section{Cytoplasmic droplets: post-ejaculatory retention and infertility}

The fact that droplet loss at ejaculation is necessary for fertility is suggested by the association of subfertility or infertility in certain transgenic infertile male mouse models in which flagellar angulation occurs at the site of the retained droplet. ${ }^{12,69,70}$ The retention of cytoplasmic droplets on ejaculated spermatozoa is also associated with infertility in bulls ${ }^{71,72}$ and boars. ${ }^{73,74}$ Interestingly, these species are among others (stallions, goats and dogs) that exhibit the Dag defect, a 
flagellar angulation characteristic of seminal spermatozoa from these infertile males. ${ }^{12,75}$ Flagellar angulation occurs at the site of the cytoplasmic droplet, as it does in the infertile mice, proving that the ejaculated spermatozoa had retained their droplets. The infertility of spermatozoa with droplets has been suggested to reflect poor adherence to the zona pellucida $\left(\mathrm{cows}^{72}\right)$ and the oviductal epithelium (sows $^{76,77}$ ). As spermatozoa with retroflected flagella swim 'backwards' (head against the direction of $\operatorname{travel}^{78}$ ), it is equally likely that their passage through mucus or the uterotubal junction is hampered and the oocyte is never reached, as is the case for the c-ros $\mathrm{KO}$ mice. ${ }^{23}$ Human spermatozoa are a special case and are discussed separately below. (It is interesting to note that some ovine testicular spermatozoa angulate at the mid-/prinicipal-piece junction when subjected to cold shock in their native rete testis fluid, although the droplet is still at the neck. ${ }^{9}$ This may reflect a structural weakness at that site, as no overt hypotonic stress is involved and RVD mechanisms are temperature independent.)

\section{SPECULATION ON A RELATIONSHIP BETWEEN CYTOPLASMIC DROPLET LOSS AND RVD}

If droplets are involved in loading and unloading spermatozoa with osmolytes, and the osmolytes are related to fertility, two related questions have to be answered: why are the droplets lost at ejaculation and why is their retention associated with infertility?

\section{Consequences of droplet loss and retention for spermatozoa in the female tract}

The distribution of osmolytes within spermatozoa and their droplets has not been addressed, but if osmolytes are accumulated within the cytoplasm in the droplet, its loss at ejaculation would deprive spermatozoa of the driving force for volume regulation. This would put in jeopardy any cell experiencing a hypotonic insult requiring an osmolyte-related RVD response. On the other hand, removal of the droplets would eliminate the high osmolality associated with the osmolytes and thus reduce any osmotic entry of water faced in the female tract, so that RVD would not necessarily be invoked. (Epididymal spermatozoa, with their retained droplets, when inseminated into the female tract would lose their droplets in the same manner as ejaculated spermatozoa when transferred to hypotonic inseminating medium.) On the other hand, the retention of an osmolyte-enriched droplet after ejaculation would encourage osmotic water entry from hypotonic female tract fluids, leading to swelling, unless rapidly countered by RVD. This would result in either flagellar bending or droplet rupture, depending on the stiffness of the flagellum, and either response would prevent sperm migration within the female tract.

From this viewpoint, droplet retention could become a liability, enforcing flagellar angulation, whereas droplet loss would maintain a straight flagellum and enable the progressive motility that permits sperm progress through the uterotubal junction or cervical mucus. From both of these viewpoints, it would be advantageous for spermatozoa to lose their droplets; indeed, elimination of the droplet at ejaculation would be prognostic for fertility and its retention diagnostic for infertility.

\section{Site of loss of the droplets: in the male}

Any removal of the droplet before, or at the time of, entry into the female tract, would occur to the droplet-bearing spermatozoa in the epididymis, vas deferens and ampulla on experiencing the hypotonic challenge of accessory gland fluids at ejaculation. In the mouse, female tract fluids are about the same osmolality as that of the seminal vesicles, ${ }^{12}$ so that the major osmotic insult to epididymal spermatozoa must occur at ejaculation.

It is speculated here that this osmotic insult is the cause of droplet removal; this is a slightly different interpretation of the data from that previously considered. Hitherto, a low sperm osmolyte load was considered to cause infertility, because inadequate RVD had led to flagellar angulation at the site of the droplet. An alternative view is raised here that inadequate RVD itself hinders droplet loss and, conversely, a high osmolyte content is necessary for droplet removal at ejaculation; it follows that the role of the epididymis in osmolyte loading is that of preparing the spermatozoa to lose their droplets rather than counteracting the consequences of retaining it. Epididymal dysfunction, associated with poor osmolyte loading of spermatozoa, would then be a direct cause of male infertility by preventing timely droplet loss. The flagellar angulation of post-coital uterine spermatozoa from c-ros $\mathrm{KO}$ mice $^{23}$ is a clear demonstration of droplet retention, and these spermatozoa are known to be swollen ${ }^{79}$ and to contain less osmolytes than those of heterozygous-type fertile males with straight sperm flagella. ${ }^{45}$

\section{Cytoplasmic droplets: possible mechanisms of loss}

The above speculation also addresses the vexing question of how the droplet is lost, as it could be intimately associated with sperm volume regulation. In the bull, seminal vesicle fluid can release droplets from testicular, mature and immature epididymal spermatozoa. ${ }^{80-82}$ In porcine semen, some spermatozoa do have droplets, but a higher percentage is found in semen from semino-vesiculectomized males, suggesting a role for accessory gland secretions in droplet loss. ${ }^{61,62} \mathrm{~A}$ haemolytic phospholipid-binding protein has been identified in the bovine ampulla and seminal vesicles, but not in epididymal fluid, which can liberate droplets from mature and immature spermatozoa from several species. ${ }^{82,83}$

Loss of the droplet must occur with a perfect resealing of the sperm's membrane at the site whence it is lost, or the loss of intracellular components necessary for sperm function (for example, ATP) would occur and immotility would result. A perfect seal is more likely if the droplet assumes a spherical shape, as this presents a minimal area at the site of constriction where droplet discharge occurs (Figure 4a). Discharge could be aided by any phospholipid-binding or other proteins in the ejaculate that modulate membrane fluidity. If osmotic entry of water into the droplet is not sufficiently countered by RVD, even a small amount of swelling may induce flagellar angulation, such that the contact area of droplets to the flagellum would be enlarged and loss of the droplet would be hampered (Figure $4 \mathrm{~b}$ ).

Such flagellar angulation would occur in the caudal spermatozoa from species with flexible flagella, such as the mouse ${ }^{79}$ and bull. ${ }^{78}$ In contrast, rat caudal spermatozoa are resistant to hypotonic flagellar bending, and droplets rupture in usual media ${ }^{26}$ unless softened by treatment with the penetrating disulphydryl bond-reducing agent dithiothreitol. ${ }^{84}$ Although freshly collected immature rat caput spermatozoa angulate easily when swollen, forming 'hairpin' and 'pigtail' bends, ${ }^{38}$ they lose their flexibility on ageing in vitro when they manage only partial angulation under hypotonic conditions, as do hamster spermatozoa. ${ }^{85}$ Flagellar oxidation in vitro is most likely the cause of this resistance to bending, and this naturally happens within the epididymis, so that cauda sperm display a rigid flagellum that favours spherical droplets ${ }^{84}$ and hence would promote droplet loss.

\section{Human cytoplasmic droplets: failure of migration and loss}

The relationship of the droplet to fertility in man differs from that in other species, but species differences have to be distinguished from 
a

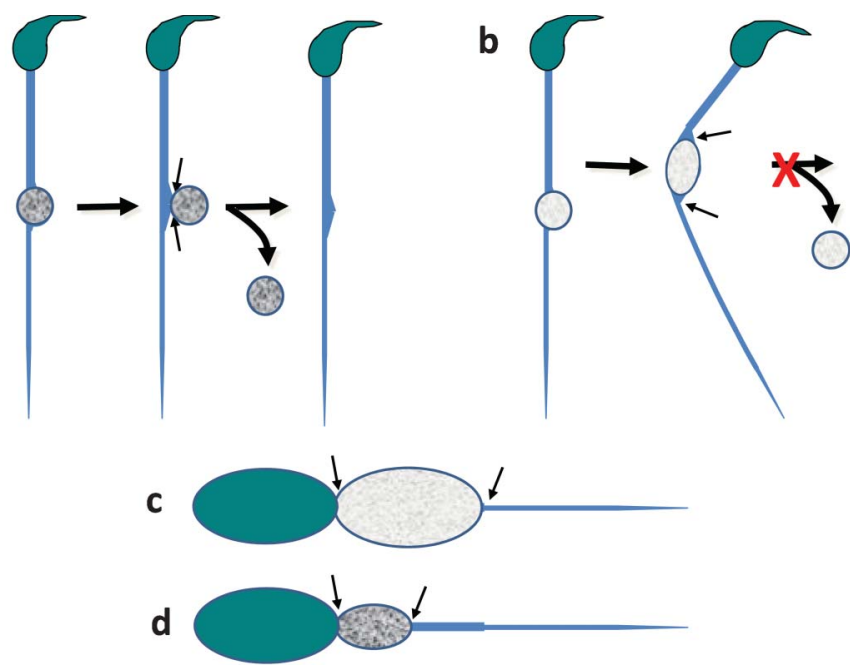

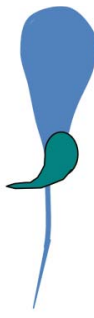

Figure 4 Schematic diagram of a postulated mechanism of loss of the sperm cytoplasmic droplet. Upper panels: rodent spermatozoon with a longer midpiece than sperm head and relatively small droplet at the annulus (not to scale). (a) With high osmolyte content (dark grey) RVD is adequate to maintain a spherical droplet form that facilitates its pinching-off (paired arrows). Such straight flagella characterize fertile males, as the spermatozoa can negotiate the uterotubal junction and progress forward in viscous mucus. (b) With a lower osmolyte content (light grey), RVD is inefficient and an asymmetrical non-spherical form develops that enforces flagellar angulation. The wide area of contact between flagellum and cytoplasmic membrane (paired arrows) prevents rounding-up, pinching-off and droplet loss. Such swollen cells characterize infertile males as the spermatozoa swim backwards and cannot negotiate the uterotubal junction or penetrate viscous mucus well. Lower panels: human spermatozoon with a midpiece as long as the sperm head and relatively large droplet at the neck (not to scale). (c) With lower osmolyte content (light grey), RVD is less efficient and cytoplasmic swelling enlarges the droplet which extends the length of the midpiece. (d) With high osmolyte content (dark grey), RVD is adequate to limit the size of the droplet so that it does not extend the length of the midpiece. In (c) and (d), the wide area of contact between flagellum and cytoplasmic membrane (paired arrows) prevents droplet loss, but there is no flagellar bending and spermatozoa still progress forward. RVD, regulatory volume decrease.

those emanating from their different origins and different preexamination treatments. Human epididymal spermatozoa, which possess droplets, ${ }^{86}$ are rarely available and the ejaculate is the major source of spermatozoa. Although the non-liquefied ejaculate can be examined for sperm motility and fixed for morphology, ${ }^{87}$ the nonuniform islands of motile spermatozoa between digesting seminal clots are difficult to analyse, and the rubbery mass of fixed seminal protein makes it impossible to assess morphology of large numbers of spermatozoa without difficulty. It is therefore routine to assess motility and morphology after liquefaction, ${ }^{88}$ although the smearing ('feathering') of semen, followed by air drying before fixing and staining, introduces the morphological artefacts discussed above. As ejaculated spermatozoa experience rising osmolality during liquefaction after a sudden decrease at ejaculation, ${ }^{89}$ the gradual osmotic changes to which the spermatozoa have been subjected by the time of examination differ markedly from the rapid changes confronting epididymal spermatozoa examined soon after collection. In addition to these procedural differences, human spermatozoa differ from those of other mammals in three ways that may be related to volume regulation.

First, the cytoplasmic droplets on normal spermatozoa (not to be confused with excess residual cytoplasm of abnormal cells) are not normally shed at ejaculation. ${ }^{61}$ When human spermiogenesis is abnormal, the cytoplasm of the round spermatid is not fully removed and an abnormal spermatozoon is shed from the germinal epithelium; it travels through the epididymis and appears in the human ejaculate as a spermatozoon with 'excess residual cytoplasm' ${ }^{88}$ It is important to be aware of the distinction between excess residual cytoplasm, which survives air drying, and cytoplasmic droplets, which do not, ${ }^{61}$ when interpreting human sperm morphology in semen smears or in wet preparations. In man, excess residual cytoplasm harbours reactive oxygen species that can damage sperm membrane lipids, proteins and DNA, and can disrupt sperm function, as shown by the association of their presence in human semen with human infertility. ${ }^{61,62}$
Second, the presence of droplets on human ejaculated spermatozoa does not appear to be disadvantageous, because they are present on living (motile) cells in semen, ${ }^{90}$ culture medium, ${ }^{87,91,92}$ cervical mucus $^{93}$ and spermatozoa recovered from the upper tract, as judged from the cytoplasmic swelling observed on the few spermatozoa depicted after recovery from the Pouch of Douglas post-coitum. ${ }^{94}$ They do not interfere with motility, as they are found on a higher percentage of motile than that of immotile spermatozoa, ${ }^{87,90}$ and they do not hinder sperm migration through surrogate mucus. ${ }^{90}$ As the droplets house osmolyte and water channels ${ }^{35,53}$ they are most likely to be the site of volume regulation, as in other species. Spermatozoa from fathers and normozoospermic men perform more effective volume regulation that those from infertile patients, as a greater proportion of the cytoplasmic volume partakes in the process. ${ }^{90,95}$

Third, the cytoplasmic droplet does not appear to migrate along the midpiece, because both testicular and ejaculated human spermatozoa have a cytoplasmic droplet behind the head (Figure 5a). The length of the human sperm midpiece $\left(\sim 4.0 \mu \mathrm{m}^{96}\right.$ approximates that of the sperm head $(\sim 4.5 \mu \mathrm{m})$. Microscopical observations of living spermatozoa in culture medium by phase contrast, differential interference and X-ray microscopy (Figure 5b-j), and fortunate ultramicrographs of well-fixed human ejaculated spermatozoa within semen $^{97}$ (Figure 5k), all demonstrate that a swollen droplet is able to extend the length of the midpiece. For other species (for example, cat, dog, ram and horse) with spermatozoa of similar tail and head dimensions as those of man, the midpiece is considerably longer than the head, ${ }^{62,63}$ and in these species droplet migration within the epididymis is observed.

\section{Human cytoplasmic droplets: speculations on droplet retention and hyperactivation}

The anchoring of the swollen droplet at the neck and the annulus (Figure 5k) represents an exaggerated condition of what was described 

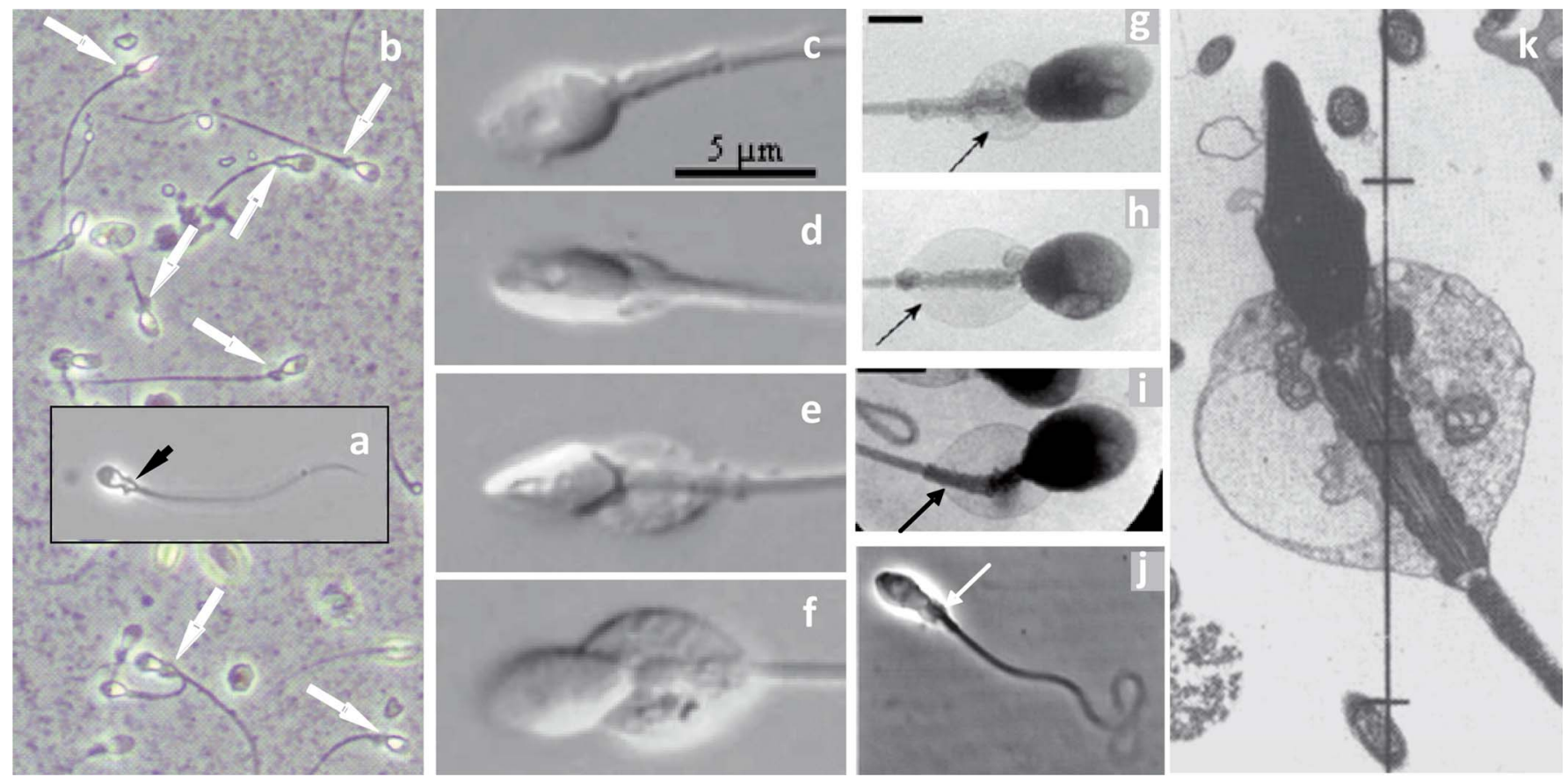

Figure 5 Montage of micrographs of (a) a human testicular spermatozoon (fixed in spermatocoele fluid), (b) human ejaculated spermatozoa (fixed in semen), both in phase contrast optics, showing the presence of cytoplasmic droplets (arrows) ('midpiece vesicles') at the neck and midpiece region (Cooper TG, unpubl. data). (c-f) Living ejaculated human spermatozoa in semen showing droplets of different size (differential contrast microscopy from Fetic et al. ${ }^{90}$ with permission), (g-i) in medium (X-ray microscopy from Chantler and Abraham-Peskir ${ }^{91}$ with permission), in cervical mucus (j) (phase contrast microscopy from Abraham-Peskir et al. ${ }^{93}$ with permission) and a section of a human spermatozoon fixed in seminal plasma (from Smith et al. ${ }^{97}$ with permission). In (k), note the expanded vesicles that may be part of the Golgi membranes. ${ }^{56}$ Droplets are located only on the midpiece and may extend along its entire length.

above for the infertile males of species in which droplet loss is the ejaculatory norm (Figure $4 \mathrm{~b}$ ), and for the same reason: it permits no spherical shape to form, restraining the droplet physically and preventing it from being pinched off (Figure 4c). This applies equally well to cells whose droplet does not extend to the end of the midpiece (Figure 4d). Whatever the extent of cytoplasmic swelling, there is no angulation or coiling of the human flagellum under physiologically hypotonic conditions and thus no hydrodynamic hindrance to sperm function caused by droplet retention. (If the osmotic challenge is far from physiological, such as that imposed in the hypo-osmotic swelling test, ${ }^{88}$ a state for which RVD cannot cope, coiling and other flagellar contortions are inevitable.)

In humans, prostatic secretions ${ }^{98}$ and preliquefied semen ${ }^{89}$ have about the same osmolality as that of female tract fluids, ${ }^{12}$ so that, as also found for the mouse, the major osmotic insult occurs at ejaculation with no further major osmotic challenge awaiting the spermatozoa deposited in the female tract. The retained droplet presumably contains sufficient osmolytes to overcome the anticipated volume challenges within seminal fluid. Treatment of spermatozoa (for example, addition of cryoprotectants) may activate RVD and initiate premature loss of osmolytes ${ }^{12}$ with consequences for subsequent sperm functions. ${ }^{99,100}$

Provided that the human sperm cell has sufficient osmolytes to regulate its volume, the motility expressed will be forward progressive, that is, motility of the nature needed for spermatozoa to enter mucus in the cervix and then swim free of it in the uterus. When human sperm volume cannot be controlled, whether or not cryoprotectants, low osmolyte loading or an osmolyte channel blocker is the cause, the cells swell, forward migration is reduced and hyperactivation-like motion occurs. ${ }^{47,101}$ Hyperactivation may be essential for spermatozoa to escape from the isthmic reservoir and to penetrate the cumulus oophorus and zona pellucida, but is inappropriate for spermatozoa within semen, cervical mucus and the uterus and lower oviduct, where progressive motion aids their advance.

It can be further speculated that the depletion of regulatory osmolytes is another and additional trigger to hyperactivation. If local conditions within the oviduct do not reflect the composition of the bulk fluids available for analysis, ${ }^{102}$ but are hyperosmolal to luminal fluids in the cryptic locality of epithelial-bound spermatozoa, then the hypoosmotic insult arriving with the follicular fluid would be a perfectly timed final trigger to osmotic swelling. Osmotically driven hyperactivation would only occur in those spermatozoa whose osmolyte reserves are inadequate for complete RVD as they approach the freshly ovulated egg. This paradigm raises the possibility that the fertilizing spermatozoon selects itself from the palette of spermatozoa arriving in the oviduct. Some spermatozoa, with too small an initial osmolyte reserve that is depleted too early, would undergo swelling-induced hyperactivation in semen or mucus and never arrive in the oviduct. Others, with more of an initial osmolyte reserve, would regulate volume and progress beyond the site of fertilization before RVD failed and hyperactivation ensued a long time after ovulation. In contrast, the fertilizing spermatozoon, losing its osmolyte load just at the time when the egg enters the oviduct, would exhibit hyperactivation at the right time and be best placed to affect fertilization. Such behaviour requires high numbers of spermatozoa in the oviduct, each of different osmolyte loads. The 'optimal' sperm osmolyte load would clearly depend on the life history of the spermatozoon (for example epididymal loading) as well as the physiological state of the female tract on sperm arrival. 


\section{COMPETING FINANCIAL INTERESTS}

The author declares no conflict of interests.

1 Yeung $\mathrm{CH}$, Cooper TG. Acquisition and development of sperm motility upon maturation in the epididymis. In: Robaire B, Hinton BT, editors. The Epididymis From Molecules to Clinical Practice. A Comprehensive Survey of the Efferent Ducts, the Epididymis and the Vas Deferens. New York: Kluwer Academic/Plenum Publishers; 2002. pp417-34.

2 Jow WW, Steckel J, Schlegel PN, Magid MS, Goldstein M. Motile sperm in human testis biopsy specimens. J Androl 1993; 14: 194-8.

3 Cooper TG, Raczek S, Yeung CH, Schwab E, Schulze H, et al. Composition of fluids obtained from human epididymal cysts. Urol Res 1992; 20: 275-80.

4 Hirsch A, Montgomery J, Mohan P, Mills C, Bekir J, et al. Fertilisation by testicular sperm with standard IVF techniques. Lancet 1993; 342: 1237-8.

5 Craft I, Tsirigotis M, Zhu JJ. In-vitro culture of testicular sperm. Lancet 1995; 346 1438.

6 Edirisinghe WR, Junk SM, Matson PL, Yovich JL. Changes in motility patterns during in vitro culture of fresh and frozen/thawed testicular and epididymal sperm implications for planning treatment by intracytoplasmic sperm injection. Human Reprod 1996; 11: 2474-6.

7 Zhu J, Tsirigotis M, Pelekanos M, Craft I. In-vitro maturation of human testicular spermatozoa. Hum Reprod 1996; 11: 231-2.

8 Liu J, Tsai YL, Katz E, Compton G, Garcia JE, et al. Outcome of in-vitro culture of fresh and frozen-thawed human testicular spermatozoa. Hum Reprod 1997; 12: 1667-72.

9 VogImayr JK, Scott TW, Setchell BP, Waites GM. Metabolism of testicular spermatozoa and characteristics of testicular fluid collected from conscious rams. J Reprod Fertil 1967; 14: 87-99.

10 Carr DW, Usselman MC, Acott TS. Effects of $\mathrm{pH}$, lactate, and viscoelastic drag on sperm motility: a species comparison. Biol Reprod 1985; 33: 588-95.

11 Pholpramool C, Zupp JL, Setchell BP. Motility of undiluted bull epididymal spermatozoa collected by micropuncture. J Reprod Fertil 1985; 75: 413-20.

12 Cooper TG, Barfield JP. Utility of infertile male models for contraception and conservation. Mol Cell Endocrinol 2006; 250: 206-11.

13 Yeung $\mathrm{CH}$, Cooper TG. Developmental changes in signalling transduction factors in maturing sperm during epididymal transit. Cell Mol Biol 2003; 49: 341-9.

14 Navarro B, Kirichok Y, Clapham DE. KSper, a pH-sensitive $\mathrm{K}^{+}$current that controls sperm membrane potential. Proc Natl Acad Sci USA 2007; 104: 7688-92.

15 Santi CM, Martínez-López P, de la Vega-Beltrán JL, Butler A, Alisio A, et al. The SLO3 sperm-specific potassium channel plays a vital role in male fertility. FEBS Lett 2010; 584: 1041-6.

16 Yeung $\mathrm{CH}$, Pérez-Sánchez F, Soler C, Poser D, Kliesch S, et al. Maturation of human epididymal spermatozoa (from selected epididymides of prostatic carcinoma patients) with respect to their morphology and ability to undergo the acrosome reaction. Human Reprod Update 1997; 3: 205-13.

17 Soler C, Pérez-Sánchez F, Schulze H, Bergmann M, Oberpenning F, et al. Objective evaluation of the morphology of human epididymal sperm heads. Int J Androl 2000; 23: 77-84.

18 Calvin HI, Bedford JM. Formation of disulphide bonds in the nucleus and accessory structures of mammalian spermatozoa during maturation in the epididymis. J Reprod Fertil 1971; 13: 65-75.

19 Bedford JM, Calvin H, Cooper GW. The maturation of spermatozoa in the human epididymis. J Reprod Fertil 1973; Suppl 18: 199-213.

20 Fornés MW, de Rosas JC. Changes in the rat sperm head during epididymal transit. Gamete Res 1989; 24: 453-9.

21 De Rosas JC, Burgos MH. Epididymal transit and morphology of the hamster sperm head. Microsc Electron Biol Celular 1987; 11: 67-79.

22 Moore H, Dvorakova K, Jenkins N, Breed W. Exceptional sperm cooperation in the wood mouse. Nature 2002; 418: 174-7.

23 Yeung $\mathrm{CH}$, Wagenfeld A, Nieschlag E, Cooper TG. The cause of infertility of male c-ros tyrosine kinase receptor knockout mice. Biol Reprod 2000; 63: 612-8.

24 Ludwig L, Frick J. Spermatology: An Atlas and Manual. Berlin: Springer Verlag; 1990.

25 Merton H. Studies on reproduction in the albino mouse. II. Contributions on the maturation of the sperm cells. Proc $R$ Soc (Edinb) 1939; 59: 145-52.

26 Cooper TG, Yeung $\mathrm{CH}$. Acquisition of volume regulatory response of sperm upon maturation in the epididymis and the role of the cytoplasmic droplet. Microsc Res Tech 2003; 61: 28-38.

27 Christova Y, James PS, Cooper TG, Jones R. Lipid diffusion in the plasma membrane of mouse spermatozoa. Changes during epididymal maturation, effects of $\mathrm{pH}$, osmotic pressure and knockout of the c-ros gene, J Androl 2002; 23: 384-92.

28 Christova Y, James P, Mackie A, Cooper TG, Jones R. Molecular diffusion in sperm plasma membranes during epididymal maturation. Mol Cell Endocrinol 2004; 216: 41-6.

29 Kato S, Yasui T, Kanda S. Migration of cytoplasmic droplet in goat testicular spermatozoa by centrifugation. Jap J Anim Reprod 1983; 29: 214-6.

30 Kato S, Yasui T, Nano I, Kanda S. Migration of cytoplasmic droplet in boar spermatozoa by centrifugation. Jap J Anim Al Res 1984; 6: 15-8.

31 Jones R. Preparation of a plasma membrane-rich fraction from rat spermatozoa J Reprod Fertil 1986; 77: 435-49.
32 Jones R, Howes E, Dunne PD, James P, Bruckbauer A, et al. Tracking diffusion of GM1 gangliosides and zona pellucida binding molecules in sperm plasma membranes following cholesterol efflux. Develop Biol 2010; 339: 398-406.

33 Sobrero AJ, MacLeod J. The immediate postcoital test. Fertil Steril 1962; 13: 184-9.

34 Cooper TG, Barfield JP, Yeung $\mathrm{CH}$. The tonicity of murine epididymal spermatozoa and their permeability towards common cryoprotectants and epididymal osmolytes. Reproduction 2008; 53: 625-33.

35 Yeung $\mathrm{CH}$, Barfield JP, Cooper TG. Physiological volume regulation by spermatozoa. Mol Cell Endocrinol 2006; 250: 98-105.

36 Schweisguth DC, Hammerstedt RH. Evaluation of plasma membrane stability by detergent-induced rupture of osmotically swollen sperm. J Biochem Biophys Methods 1992; 24: 81-94.

37 Druart X, Gatti JL, Huet S, Dacheux JL, Humblot P. Hypotonic resistance of boar spermatozoa: sperm subpopulations and relationship with epididymal maturation and fertility. Reproduction 2009; 137: 205-13.

38 Cooper TG. Osmotic swelling of maturing rat spermatozoa and lysis of caput spermatozoa by acylcarnitine and acylcholines. Gamet Res 1986; 14: 47-56.

39 McClean R, MacCallum C, Blyde D, Holt WV, Johnston SD. Ultrastructure, osmotic tolerance, glycerol toxicity and cryopreservation of caput and cauda epididymidal Kangaroo spermatozoa. Reprod Fertil Dev 2006; 18: 469-76.

40 Sahin E, Petrunkina AM, Waberski D, Harrison RA, Töpfer-Petersen E. Control of bul sperm cell volume during epididymal maturation. Reprod Fertil Dev2009; 21: 469-78.

41 Sahin E, Petrunkina AM, Ekhlasi-Hundrieser M, Hettel C, Waberski D, et al. Fibronectin type II-module proteins in the bovine genital tract and their putative role in cell volume control during sperm maturation. Reprod Fertil Dev 2009; 21 479-88.

42 Damm OS, Cooper TG. Maturation of sperm volume regulation in the rat epididymis. Asian J Androl 2010; 12: 578-90.

43 Yeung $\mathrm{CH}$, Anapolski M, Sipila P, Wagenfeld A, Poutanen M, et al. Sperm volume regulation: maturational changes in fertile and infertile transgenic mice and association with kinematics and tail angulation. Biol Reprod 2002; 67: 269-75.

44 Yeung $\mathrm{CH}$, Barfield JP, Anapolski M, Cooper TG. Volume regulation of mature and immature spermatozoa in a primate model, and possible ion channels involved. Hum Reprod 2004; 19: 2587-93.

45 Yeung $\mathrm{CH}$, Anapolski M, Setiawan I, Lang F, Cooper TG. Effects of putative epididymal osmolytes on sperm volume regulation of fertile and infertile c-ros transgenic mice. $J$ Androl 2004; 25: 216-23.

46 Hinton BT, Pryor JP, Hirsh AV, Setchell BP. The concentration of some inorganic ions and organic compounds in the luminal fluid of the human ductus deferens. Int J Androl 1981; 4: 457-61.

47 Yeung CH, Anapolski M, Depenbusch M, Zitzmann M, Cooper TG. Human sperm volume regulation. Response to physiological changes in osmolality, channel blockers and potential sperm osmolytes. Hum Reprod 2003; 18: 1029-36.

48 Kulkarni SB, Sauna ZE, Somlata V, Sitaramam V. Volume regulation of spermatozoa by quinine-sensitive channels. Mol Reprod Dev 1997; 46: 535-50.

49 Turner TT. Necessity's potion: inorganic ions and small organic molecules in the epididymal lumen. In: Robaire B, Hinton BT, editors. The Epididymis. From Molecules to Clinical Practice. A Comprehensive Survey of the Efferent Ducts, the Epididymis and the Vas Deferens. New York: Kluwer Academic/Plenum; 2002. pp131-50.

50 Jeulin C, Dacheux JL, Soufir JC. Uptake and release of free L-carnitine by boar epididymal spermatozoa in vitro and subsequent acetylation rate. J Reprod Fertil 1994; 100: 263-71.

51 Pruneda A, Yeung CH, Bonet S, Pinart E, Cooper TG. Concentrations of carnitine, glutamate and myo-inositol in epididymal fluid and spermatozoa from boars. Anim Reprod Sci 2006; 97: 344-55.

52 Kobayashi D, Tamai I, Sai Y, Yoshida K, Wakayama T, et al. Transport of carnitine and acetylcarnitine by carnitine/organic cation transporter (OCTN) 2 and OCTN3 into epididymal spermatozoa. Reproduction. 2007; 134: 651-8.

53 Yeung $\mathrm{CH}$, Callies C, Rojek A, Nielsen S, Cooper TG. Aquaporin isoforms involved in physiological volume regulation of murine spermatozoa. Biol Reprod 2009; 80: 350-7.

54 Yeung $\mathrm{CH}$, Cooper TG. Aquaporin AQP11 in the testis: molecular identity and association with the processing of residual cytoplasm of elongated spermatids. Reproduction 2010; 139: 209-16.

55 Yeung $\mathrm{CH}$, Callies C, Tüttelmann F, Kliesch S, Cooper TG. Aquaporins in the human testis and spermatozoa-identification, involvement in sperm volume regulation and clinical relevance. Int J Androl 2010; 33: 629-41.

56 Oko R, Hermo L, Chan PT, Fazel A, Bergeron JJ. The cytoplasmic droplet of rat epididymal spermatozoa contains saccular elements with Golgi characteristics. J Cell Biol 1993; 123:809-21.

57 Wagenfeld A, Yeung $\mathrm{CH}$, Shivaji S, Sundareswaran VR, Aragi $\mathrm{H}$, et al. Expression and cellular localization of contraception-associated protein 1. J Androl 2000; 21: 954-63.

58 Sipilä P, Cooper TG, Yeung CH, Mustonen M, Pentinnen J, et al. Epididymal dysfunction initiated by the expression of Simia Virus $40 \mathrm{~T}$-antigen leads to angulated flagella and infertility in transgenic mice. Mol Cel Endocrinol 2002; 16 : 2603-17.

59 Temple-Smith PD. Phagocytosis of sperm cytoplasmic droplets by a specialized region in the epididymis of the brushtailed possum, Trichosaurus vulpecula. Biol Reprod 1984; 30: 707-20.

60 Hermo L, Dworkin J, Oko R. Role of epithelial clear cells of the rat epididymis in the disposal of the contents of cytoplasmic droplets detached from spermatozoa. Am J Anat 1988; 183:107-24. 
61 Cooper TG. Cytoplasmic droplets: the good, the bad or just confusing? Hum Reprod 2005; 20: 9-11.

62 Cooper TG. Sperm cytoplasmic droplets and ART. Embryo Talk 2006; 1.3: 129-36.

63 Cooper TG. The human epididymis, sperm maturation and storage. ANIR-ANHP 2007; 9: 18-24.

64 White IG, Larsen LH, Wales RG. Method for the in vivo collection of epididymal spermatozoa and for their comparison with ejaculated cells. Fertil Steril 1959; 10: $571-7$.

65 Lasley JF, Bogart R. Some factors affecting the resistance of ejaculated and epididymal spermatozoa of the boar to different environmental conditions. Am J Physiol 1944; 141: 619-24.

66 Lasley JF, Bogart R. A comparative study of epididymal and ejaculated spermatozoa of the boar. J Anim Sci 1944; 3: 360-70.

67 O'Donnell JM. Electrical counting and sizing of mammalian spermatozoa and cytoplasmic droplets. J Reprod Fertil 1969; 19: 263-72.

68 Kato S, Shibukawa T, Harayama H, Kanna Y. Timing of shedding and disintegration of cytoplasmic droplets from boar and goat spermatozoa. J Reprod Develop (Tokyo) 1996; 42: 237-241.

69 Cooper TG, Yeung CH, Wagenfeld A, Nieschlag E, Poutanen M, et al. Mouse models of infertility due to swollen spermatozoa. Mol Cell Endocrinol 2004; 216: 55-63.

70 Hinton BT, Cooper TG. The epididymis as a target for male contraceptive development. In: Habenicht U-F, Aitken JA, editors. Fertility Control-Today and in the Future. Berlin: Springer Press; 2010.

71 Amann RP, Seidel GE, Mortimer RG. Fertilizing potential in vitro of semen from young beef bulls containing a high or low percentage of sperm with a proximal droplet. Theriogenology 2000; 54: 1499-1515.

72 Thundathil J, Palasz AT, Barth AD, Mapletoft RJ. The use of in vitro fertilization techniques to investigate the fertilizing ability of bovine sperm with proximal droplets. Anim Reprod Sci 2001; 65: 181-92.

73 Kuster CE, Hess RA, Althouse GC. Immunofluorescence reveals ubiquitination of retained distal cytoplasmic droplets on ejaculated porcine spermatozoa. J Androl 2004; 25: 340-7.

74 Waberski D, Meding S, Dirksen G, Weitze KF, Leiding C, et al. Fertility of long-termstored boar semen: influence of extender (Androhep and Kiev), storage time and plasma droplets in the semen. Anim Reprod Sci 1994; 36: 145-51.

75 Cooper TG. The epididymis as a site of contraceptive attack. In: Nieschlag E, Habenicht UF, editors. Spermatogenesis, Fertilization, Contraception. Molecular, Cellular and Endocrine Events in Male Reproduction. Berlin: Springer Verlag; 1992. pp419-60.

76 Petrunkina AM, Gehlhaar R, Drommer W, Waberski D, Töpfer-Petersen E. Selective sperm binding to pig oviductal epithelium in vitro. Reproduction 2001; 121: 889-96.

77 Khalil AA, Petrunkina AM, Sahin E, Waberski D, Töpfer-Petersen E. Enhanced binding of sperm with superior volume regulation to oviductal epithelium. J Androl 2006; 27: 754-65

78 Phillips DM, Kalay D. Mechanisms of flagellar motility deduced from backwardswimming bull sperm. J Exp Zool 1984; 231: 109-16.

79 Yeung $\mathrm{CH}$, Anapolski M, Cooper TG. Measurement of volume changes in mouse spermatozoa using an electronic sizing analyzer and a flow cytometer: validation and application to an infertile mouse model. J Androl 2002; 23: 522-8.

80 Bialy G. Smith VR. Influence of seminal vesicular fluid on morphology of bull spermatozoa. J Dairy Sci 1958; 41: 422-8.

81 Larson RE, Crabo B, Leman AD. Physical and chemical influences on loss of the cytoplasmic droplet (CD) from porcine spermatozoa during ejaculation. Proceedings of the 9th International Congress on Animal Reproduction and Artificial Insemination; 16-20 June 1980; Madrid, Spain. Ministerio Agricultura: Madrid; 1980. Vol. 3, pp45-8.
82 Matousek J, Kysilka C. The phospholipid-binding protein of the reproductive tract of the bull-effect on the removal of spermatozoal cytoplasm droplets in other species and influence of antibodies on its reactivity. Anim Reprod Sci 1984; 7: 433-40.

83 Matousek J, Kysilka C. The haemolytic factor (phospholipid-binding protein) of the bull reproductive tract-its synthesis and effect on spermatozoal cytoplasm droplets. Anim Reprod Sci 1989; 3: 195-205.

84 Cooper TG. Prevention of hypo-osmotic swelling by detergents provides clues to the membrane structure of rat sperm. Int J Androl 1985; 8: 159-67.

85 Yeung $\mathrm{CH}$, Oberländer G, Cooper TG. Maturation of hamster epididymal sperm motility and influence of the thiol status of hamster and rat spermatozoa on their motility patterns. Mol Reprod Dev 1994; 38: 347-55.

86 Ånberg $\AA$. The ultrastructure of the human spermatozoon; an electronmicroscopic study of the spermatozoa from sperm samples and the epididymis including some observations of the spermatid. Acta Obstet Gynecol Scand Supp/ 1957; 36(Suppl 2): $1-133$

87 Cooper TG, Yeung CH, Fetic S, Sobhani A, Nieschlag E. Cytoplasmic droplets are normal structures of human sperm but are not well preserved by routine procedures for assessing sperm morphology. Hum Reprod 2004; 19: 2283-8.

88 World Health Organization. WHO Laboratory Manual for the Examination and Processing of Human Semen. 5th ed. Geneva: WHO Press; 2010.

89 Cooper TG, Barfield JP, Yeung CH. Changes in osmolality during liquefaction of human semen. Int J Androl 2005; 28: 58-60.

90 Fetic S, Yeung CH, Sonntag B, Nieschlag E, Cooper TG. Relationship of cytoplasmic droplets to motility, migration in mucus, and volume regulation of human spermatozoa. J Androl 2006; 27: 294-301.

91 Chantler E, Abraham-Peskir JV. Significance of midpiece vesicles and functional integrity of the membranes of human spermatozoa after osmotic stress. Andrologia 2004; 36: 87-93.

92 Lishko PV, Botchkina IL, Fedorenko A, Kirichok Y. Acid extrusion from human spermatozoa is mediated by flagellar voltage-gated proton channel. Cell 2010; 140: 327-37.

93 Abraham-Peskir JV, Chantler E, Uggerhoj E, Fedder J. Response of midpiece vesicles on human sperm to osmotic stress. Hum Reprod 2002; 17: 375-82.

94 Mortimer D, Leslie EE, Kelly RW, Templeton AA. Morphological selection of human spermatozoa in vivo and in vitro. J Reprod Fertil 1982; 64: 391-9.

95 Yeung $\mathrm{CH}$, Cooper TG. Potassium channels involved in human sperm volume regulation-quantitative studies at the protein and mRNA levels. Mol Reprod Dev 2008; 75: 659-68.

96 Cummins JM, Woodall PF. On mammalian sperm dimensions. J Reprod Fertil 1985; 75: 153-75.

97 Smith AC, Anderson CW, Barratt CL, Williams MA. Ultrastructural morphometric data on human spermatozoa. Andrologia 1988; 20: 396-403.

98 Björndahl L, Kvist U. Sequence of ejaculation affects the spermatozoon as a carrier and its message. Reprod Biomed Online 2003; 7: 440-8.

99 Widiasih D, Yeung CH, Junaidi A, Cooper TG. Multistep and single-step treatment of human spermatozoa with cryoprotectants. Fertil Steril 2009; 92: 382-9.

100 Setyawan EE, Cooper TG, Widiasih DA, Junaidi A, Yeung CH. Effects of cryoprotectant treatments on bovine sperm function and osmolyte content. Asian J Androl 2009; 11: $571-81$

101 Yeung $\mathrm{CH}$, Cooper TG. Effects of the ion-channel blocker quinine on human sperm volume, kinematics and mucus penetration, and the involvement of potassium channels. Mol Hum Reprod 2001; 7: 819-28.

102 Hunter RH. The Fallopian tubes in domestic mammals: how vital is their physiological activity? Reprod Nutr Dev 2005; 45: 281-90. 